\title{
Use of Lasers in the Treatment of Alopecia Areata
}

\author{
Howard Chu \\ Do Young Kim
}

Department of Dermatology, Severance Hospital, Cutaneous Biology Research Institute, Yonsei University College of Medicine, Seoul, Korea
Received October 28, 2016

Revised November 26, 2016

Accepted November 27, 2016

\section{Correspondence}

Do Young Kim

Department of Dermatology and Cutaneous Biology Research Institute, Yonsei University College of Medicine, 50-1 Yonsei-ro, Seodaemungu, Seoul 03722, Korea

Tel.: +82-2-2228-2080

Fax: +82-2-393-9157

E-mail: dykimayuhs.ac

(C) Korean Society for Laser Medicine and Surgery

(c) This is an open access article distributed under the terms of the Creative Commons Attribution NonCommercial License (http://creativecommons.org/ licenses/by-nc/4.0) which permits unrestricted noncommercial use, distribution, and reproduction in any medium, provided the original work is properly cited.
Alopecia areata (AA) is a complex autoimmune disease characterized by round or oval patches of non-scarring hair loss that is mediated by lymphocytes affecting the hair follicles. To treat localized AA, intralesional corticosteroid injections and topical corticosteroids are most commonly used, while for widespread AA, systemic steroids, immunosuppresants, and contact immunotherapy are commonly considered. However, therapeutic options for refractory AA that is unresponsive to these conventional treatments are limited. Various lasers have been suggested in recent studies to treat AA. The effect of 308-nm excimer laser was the most studied, while others, including neodymium-doped yttrium aluminum garnet, erbium:glass laser, fractional CO2 laser, and low-level laser therapy, have also been assessed. Despite the nascent state of research on lasers as a treatment option for $A A$, there have been a great deal of promising results. It is possible for lasers to become the mainstay treatment option of AA.

\section{Key words}

Alopecia areata; Excimer laser; Laser; Paradoxical hypertrichosis 


\section{INTRODUCTION}

Alopecia areata (AA) is a complex autoimmune disease characterized by round or oval patches of non-scarring hair loss, which is mediated by lymphocytes that affects the hair follicles. 'The development of hair loss involves aberrant modulation of the hair growth cycle, resulting in dystrophic anagen hair shedding and/or increased telogen state follicles. ${ }^{2,3}$ The management of AA varies widely among dermatologists. Intralesional corticosteroid injections and topical corticosteroids are most commonly used, while topical minoxidil and topical immunotherapy are also treatment options generally considered. ${ }^{4,5}$ The treatment of refractory AA is generally considered difficult and mostly ineffective, which eventually lead to the recent studies with the use of targeted therapies. ${ }^{6,7}$ No treatments, however, have been shown to have long-term benefit compared to placebo. ${ }^{4}$

The use of lasers as another treatment option for AA began not long ago, with the observation of "paradoxical hypertrichosis," which was initially used to describe paradoxical hair growth in patients treated for photoepilation with intense pulsed light therapy. ${ }^{8}$ Since then, additional cases of paradoxical hypertrichosis have been reported, which was found to occur possibly in low fluences and all types of lasers. ${ }^{9}$ Studies have tried to investigate the underlying mechanisms, in which low fluence of heat causing sub-therapeutic injury was thought to induce follicular stem cell proliferation and differentiation by increasing the level of heat shock proteins and other factors, which may play a role in regulation of cell growth and differentiation. ${ }^{10,11}$

The basis for the use of laser had also been supported by the finding of hair growth stimulation in the process of wound healing. ${ }^{12}$ Hair follicles have been found to develop anew after wounding, via activation of Wnt-mediated signaling pathway. Conversely, cutaneous wound healing also substantially accelerates during anagen stage of hair cycle. ${ }^{13}$ Ultimately, laser treatment has been shown to induce hair regrowth by induction and prolongation of anagen through Wnt/B-catenin pathway in the murine model, ${ }^{14}$ providing evidences of the use of lasers in AA. In addition, because AA is known as an autoimmune disorder, ${ }^{15}$ lasers that may induce immunomodulatory effects, such as 308-nm excimer laser, ${ }^{16}$ has also been suggested for the treatment of AA.

The exact mechanisms of the therapeutic effects of lasers on AA are still unknown, yet studies on the use of lasers in the treatment of AA have been steadily increased. Although not widely used compared to other treatment modalities, various laser therapies had shown therapeutic efficacy and their use as another option for the treatment of refractory AA have been suggested. ${ }^{17}$

\section{8-NM EXCIMER LASER}

Excimer laser consists of xenon and chloride that emits its total energy at a wavelength of $308 \mathrm{~nm}$, which lies within the UVB spectrum. ${ }^{18,19}$ The pathophysiology underlying excimer lasers that induce the therapeutic effects in AA are thought to be different from that of other lasers. Although the exact mechanism is unclear, excimer laser is thought to induce apoptosis of T cells, thereby leading to therapeutic effects in inflammatory diseases, such as psoriasis. ${ }^{16}$ With the discovery of AA as an autoimmune disorder with T cell-mediated inflammation of the hair follicle, ${ }^{15}$ the use of excimer lasers has been suggested. Since the benefit of the excimer laser on AA first reported by Gundogan et al.., ${ }^{20}$ numerous studies have confirmed its efficacy. ${ }^{21-23}$

The largest study so far was done on 18 patients with recalcitrant $A A$ that involved the scalp, beard, arms and legs. Each alopecic patch was treated twice a week for a maximum of 24 sessions. Hair regrowth has been shown in $41.5 \%$ of the patches, whereas no growth of hair was observed in the control patches, ${ }^{22}$ confirming the efficacy of the treatment. Another study has been done on 11 children with recalcitrant $A A$ of the scalp, in which regrowth was noted in $60 \%$ of patches treated with the excimer laser for 12 weeks versus no regrowth in untreated control patches, ${ }^{24}$ suggesting that excimer lasers are safe and effective not only in adults with refractory AA, but in children as well. Recently, a comparative study was done by dividing a single alopecic patch into control and treated side and applying the excimer laser for 12 weeks, twice a week. ${ }^{25}$ The diameter of the hair was found to be significantly increased in the treated area when compared with the control side. Based on the results of the efficacy in treating refractory AA in these studies, the results suggest excimer laser as another option for the treatment of AA.

The protocols of excimer laser therapy used were generally consistent in the studies, in which the initial dose was $50 \mathrm{~mJ} / \mathrm{cm}^{2}$ less than the minimal erythema dose and the doses were then increased by $50 \mathrm{~mJ} / \mathrm{cm}^{2}$ every two session for usually every week, since the treatments were usually done twice a week. ${ }^{22,25}$ Excimer laser is a device widely used in the field of dermatology and has been considered to be relatively safe, with possible adverse events of burn, persistent erythema and pain. ${ }^{25}$ In these studies, 
excimer laser treatment on AA was generally mild and tolerable, even in children, ${ }^{24}$ in which the adverse events were mild, including erythema, hyperpigmentation, itching, and mild peeling of the skin. ${ }^{22}$

Although all studies have reported the efficacy of excimer laser in AA, the therapeutic results seem to be poor in patients with alopecia universalis and alopecia totalis, ${ }^{21}$ in which no hair regrowth was observed in patches on the extremities. ${ }^{22}$ Also, atopic diathesis had been found to a poor prognostic factor, as patients with atopic diseases had shown minimal responses to the therapy. ${ }^{22}$ Based on these findings, excimer laser can be a safe and effective therapeutic method for refractory AA confined to the scalp. The sample sizes of the previous studies, however, were small, in which large-scale clinical trials are needed to confirm the efficacy and safety of excimer lasers in the treatment of patients with $A$ A.

\section{FRACTIONAL NON-ABLATIVE PHOTOTHERMOLYSIS LASER}

Stimulation of hair growth during the wound healing process is another finding that can be the basis of laser treatment in hair regrowth ${ }^{12}$. Fractional non-ablative photothermolysis is a laser technique that produces columns of "microthermal treatment zones (MTZs)" that extend down to the reticular dermis, sparing the surrounding tissues, and as a result, does not damage the stratum corneum. ${ }^{26}$ As a result, wound-healing process would be induced, which would hypothetically cause hair growth.

The efficacy of fractional non-ablative photothermolysis laser was first observed in a report of a 35-year-old male with 2-year history of recalcitrant $A A^{27}$ who was nonresponsive to treatments with topical corticosteroids, intralesional corticosteroid injections, and topical minoxidil. The patient was treated with fractional erbium glass laser weekly for 24 weeks with a pulse energy of 10-15 mJ, a density of $300 \mathrm{MTZ} / \mathrm{cm}^{2} /$ pass, and two passes per session. Hair growth was noted after 1 month and complete regrowth was achieved at 6 months, with no hair loss in the 6-month follow-up period. A recent study has also reported the efficacy of erbium glass laser in five patients with $A A^{28}{ }^{28}$ The patients were treated for 2 to 3 sessions, with an interval of 3-6 weeks and the parameters of fluences ranging from $30-45 \mathrm{~mJ} / \mathrm{cm}^{2}, 6-8$ density and $8-10$ passes. All patients obtained fully or almost fully regrowth at 3 months, without any side effects except for minimal pain during the treatment. ${ }^{28}$

The possible mechanisms hypothesized for fractional photothermolysis laser in hair regrowth are the induc- tion of T-cell apoptosis and direct enhancement of hair growth. ${ }^{27}$ In addition, the therapeutic effects of the laser have been proven in larger number of patients with androgenic alopecia, ${ }^{29,30}$ but since the exact mechanisms of hair regrowth is unknown, the elucidation of the pathophysiology is necessary. Although the efficacy has been observed in few cases, further studies with larger sample sizes are needed to suggest fractional photothermolysis laser as a possible therapeutic option for AA in the future.

\section{LASERS USED TO INCREASE DRUG DELIVERY}

Studies have assessed the usefulness of laser therapies in increasing the delivery of the topical medications used in $\mathrm{AA}^{31,32}$ Efficient topical drug delivery may be challenging due to the barrier function of the stratum corneum and laser treatment can precisely and selectively remove the stratum corneum in a controlled and noncontact manner. ${ }^{33}$ A study was performed on nude mouse and porcine skin to evaluate whether erbium:yttrium aluminum garnet (erbium:YAG) laser may facilitate the permeability of topical medications used in alopecia. The application of the laser at an ablation depth of 6 and $10 \mu \mathrm{m}$ was found to increase the skin accumulation of minoxidil twofold to ninefold, depending on the selected skin barriers. ${ }^{32}$ A similar clinical study was performed in five patients with AA by using either radiofrequency (RF) or fractional $\mathrm{CO} 2$ lasers followed by topical application of triamcinolone, in addition to the application of acoustic pressure wave ultrasound afterwards. Complete recovery was observed in all patients with one to six sessions of treatment, depending on the clinical responses. ${ }^{31}$ Besides the ablative effect of removing the stratum corneum, the laser may interact with sebum to break up the barrier function, increasing the skin delivery of drugs. The minimally invasive, wellcontrolled approach of laser-mediated drug permeation may offer a potential way to treat alopecia. ${ }^{32}$

\section{LOW-LEVEL LASER THERAPY}

The idea of low-level laser therapy (LLLT) for the treatment of hair loss began in 1967 with the observation of increased hair growth while investigating whether cancer can be induced by low-powered ruby laser therapy 1694 $\mathrm{nm}$ ). LLLT was applied on to the shaved skin of mice, in which faster hair regrowth was observed when compared to the untreated mice. ${ }^{34}$ Several recent studies have then evaluated the efficacy of LLLT in the treatment of different types of hair loss, especially in androgenetic alopecia, in which the data have shown promising results. ${ }^{35}$ 
HairMax LaserComb (Lexington International, Boca Raton, FL, USA), a 655-nm LLLT device, which is approved by the FDA to treat androgenetic alopecia, ${ }^{36}$ was used to assessed the efficacy in a $\mathrm{C} 3 \mathrm{H} / \mathrm{HeJ}$ mouse model of $\mathrm{AA}$ induced by heat. ${ }^{10}$ After treatment of 20 seconds daily, three times per week for a total of 6 weeks, hair regrowth was observed in the mice in the treated group compared to no growth in the untreated group, with increased number of anagen hair follicles in the treated group..$^{10} \mathrm{~A}$ similar study of LaserComb used in spontaneous and graftinduced $\mathrm{AA}$ in $\mathrm{C} 3 \mathrm{H} / \mathrm{HeJ}$ mice, however, did not induce hair growth with the similar protocols, ${ }^{37}$ in which further studies are needed to settle the controversies.

Several clinical studies of LLLT have been done on androgenic alopecia with favorable outcomes, ${ }^{38}$ whereas the number of clinical studies in AA is limited. A study in 2006 has reported the effectiveness of low-level pulsed infrared diode laser $(904 \mathrm{~nm})$ in the treatment of 16 patients with 34 AA patches non-responsive to conventional treatment modalities. ${ }^{39}$ The patients received four treatments on a weekly basis, for a total of one month, with parameters of $04 / \mathrm{s}, 1.2 \mathrm{~mW}$. The success rate was reported to be very high with regrowth observed in $94 \%$ of the patches, while no regrowth was seen in the control patches. ${ }^{39} \mathrm{~A}$ similar study was recently performed with pulsed infrared diode laser on 23 patients with 52 AA patches resistant to conventional treatment modalities. ${ }^{40}$ Favorable outcomes were also observed, in which significant hair regrowth was seen in the treated patches compared to the control patches. Lastly, although technically not a laser, another study was done with linear polarized infrared light with frequencies of 600-1600 nm irradiated on 15 patients with AA for 3 minutes once every one or two weeks. ${ }^{41}$ Of the irradiated areas, $46.7 \%$ had shown hair regrowth 1.6 months earlier in the irradiated areas than in the nonirradiated areas.

The exact mechanisms of LLLT is unknown, yet it is assumed to stimulate anagen re-entry in telogen hair follicles, prolong duration of anagen phase, increase rates of proliferation in active anagen hair follicles and to prevent premature catagen development. ${ }^{10,36}$ The number of studies are scarce and the mechanisms are needed to be elucidated, but with further larger studies in the future, LLLT may be considered as a treatment option.

\section{MISCELLANEOUS LASERS}

Recently, a study has been conducted to evaluate the efficacy of two different lasers, neodymium-doped:yttrium aluminum garnet (Nd:YAG) and fractional CO2 laser, in

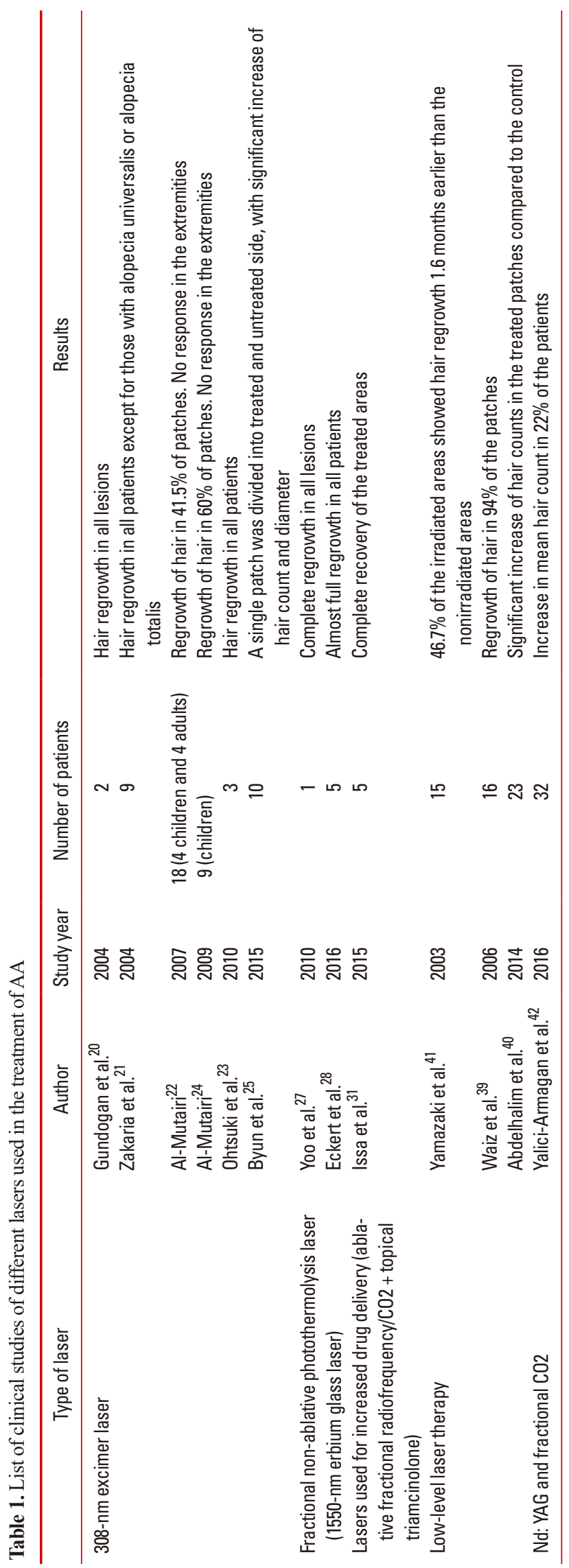


the treatment of patients with refractory AA. ${ }^{42}$ Thirtytwo patients were recruited and three different alopecic patches were selected in each patients, where $\mathrm{Nd}: Y A G$ laser was applied to one of the patches, fractional CO2 laser to the second patch, and the remaining patch was set as the control. The Nd:YAG laser was used with a pulse duration of 30 milliseconds and an energy of $10 \mathrm{~J} / \mathrm{cm}^{2}$, whereas the fractional $\mathrm{CO} 2$ laser was applied with a power of $30 \mathrm{~W}, 120 \mathrm{~mm}$ probe diameter, pulse energy range of $10-45 \mathrm{~mJ} / \mathrm{cm}^{2}$ and density range of $75-100 \mathrm{spot} / \mathrm{cm}^{2} /$ pass. The Severity of Alopecia Tool (SALT) score was used for assessment, which was found to be improved in 22\% of the patients and no statistically difference was found in the mean hair count of the three different patches in each patients. ${ }^{42}$ The use of the two lasers were not effective in the study, and the need for larger studies with different parameters of the lasers for the treatment of AA has been suggested.

\section{CONCLUSION}

Although some poor prognostic factors are known in $A A$, the course of the disease is generally unpredictable and the treatment response can be variable. ${ }^{3}$ Numerous treatment options are available and studies have found that up to $50 \%$ of patients will recover within 1 year even without treatment, ${ }^{43}$ but possible therapeutic options in refractory $A A$ are limited and the efficacies are controversial. ${ }^{4,43}$ Studies of the use of lasers in the treatment of AA have increased recently, and although some of the outcomes had been variable, most of the results had shown favorable therapeutic outcomes, ${ }^{17}$ as summarized in Table 1.

The pathophysiology of the lasers that take effect on hair regrowth in AA is still largely unknown and the mechanisms are thought to be different among the types of lasers. Excimer lasers have been studied the most, which is thought to cause hair regrowth by inducing apoptosis of T cells, ${ }^{19}$ as AA is known to be a T cell mediated disorder. ${ }^{15}$ Other lasers that have been studied include erbium glass laser, Nd: YAG laser, fractional CO2 laser, and LLLT, which have used based on the findings of paradoxical hypertrichosis and hair growth upon wound healing. ${ }^{9.12}$ In addition, some lasers have been used in order to increase the skin permeability of drugs used in alopecia. ${ }^{31,32}$ Because the sample size of the studies was small, further randomized clinical trials on larger number of patients are needed. The Korean national insurance does not cover for the use of lasers in the treatment of $A A$, hence limiting its uses in Korea. Thus, the verification of the efficacy through further studies may provide increased opportunities for the patients to receive these laser treatments in the future. Despite the limitation in the uses and the variability of the mechanisms of the different laser devices, most of the studies have shown satisfactory results, in which the use of lasers can be suggested as another therapeutic option in the treatment of $A A$.

\section{REFERENCES}

1. Hordinsky MK. Overview of alopecia areata. J Investig Dermatol Symp Proc 2013;16:S13-5.

2. McElwee KJ, Gilhar A, Tobin DJ, Ramot Y, Sundberg JP, Nakamura $M$, et al. What causes alopecia areata? Exp Dermatol 2013;22:609-26.

3. Alkhalifah A, Alsantali A, Wang E, McElwee KJ, Shapiro J. Alopecia areata update: part I. Clinical picture, histopathology, and pathogenesis. J Am Acad Dermatol 2010;62:177-88.

4. Alkhalifah A, Alsantali A, Wang E, McElwee KJ, Shapiro J. Alopecia areata update: part II. Treatment. J Am Acad Dermatol 2010;62:191-202, quiz 203-4.

5. Shapiro J. Current treatment of alopecia areata. J Investig Dermatol Symp Proc 2013;16:S42-4.

6. Biran R, Zlotogorski A, Ramot Y. The genetics of alopecia areata: new approaches, new findings, new treatments. J Dermatol Sci 2015;78:11-20.

7. Hordinsky MK. Current treatments for alopecia areata. J Investig Dermatol Symp Proc 2015;17:44-6.

8. Moreno-Arias G, Castelo-Branco C, Ferrando J. Paradoxical effect after IPL photoepilation. Dermatol Surg 2002;281:1013-6.

9. Desai S, Mahmoud BH, Bhatia AC, Hamzavi IH. Paradoxical hypertrichosis after laser therapy: a review. Dermatol Surg 2010;36:291-8.

10. Wikramanayake TC, Rodriguez R, Choudhary S, Mauro LM, Nouri K, Schachner LA, et al. Effects of the Lexington LaserComb on hair regrowth in the $\mathrm{C} 3 \mathrm{H} / \mathrm{HeJ}$ mouse model of alopecia areata. Lasers Med Sci 2012;27:431-6.

11. Bouzari N, Firooz AR. Lasers may induce terminal hair growth. Dermatol Surg 2006;32:460.

12. Ito M, Yang Z, Andl T, Cui C, Kim N, Millar SE, et al. Wntdependent de novo hair follicle regeneration in adult mouse skin after wounding. Nature 2007;447:316-20.

13. Ansell DM, Kloepper JE, Thomason HA, Paus R, Hardman MJ. Exploring the "hair growth-wound healing connection": anagen phase promotes wound re-epithelialization. J Invest Dermatol 2011;131:518-28.

14. Bae JM, Jung HM, Goo B, Park YM. Hair regrowth through wound healing process after ablative fractional laser treatment in a murine model. Lasers Surg Med 2015;47:433-40.

15. Madani S, Shapiro J. Alopecia areata update. J Am Acad 
Dermatol 2000;42:549-66.

16. Feldman SR, Mellen BG, Housman TS, Fitzpatrick RE, Geronemus RG, Friedman PM, et al. Efficacy of the 308$\mathrm{nm}$ excimer laser for treatment of psoriasis: results of a multicenter study. J Am Acad Dermatol 2002;46:900-6.

17. Rangwala S, Rashid RM. Alopecia: a review of laser and light therapies. Dermatol Online J 2012;18:3

18. McMichael AJ. Excimer laser: a module of the alopecia areata common protocol. J Investig Dermatol Symp Proc 2013;16:S779.

19. Beggs S, Short J, Rengifo-Pardo M, Ehrlich A. Applications of the Excimer Laser: A Review. Dermatol Surg 2015;41:1201-11.

20. Gundogan C, Greve B, Raulin C. Treatment of alopecia areata with the 308-nm xenon chloride excimer laser: case report of two successful treatments with the excimer laser. Lasers Surg Med 2004;34:86-90.

21. Zakaria W, Passeron T, Ostovari N, Lacour JP, Ortonne JP. 308-nm excimer laser therapy in alopecia areata. J Am Acad Dermatol 2004;51:837-8.

22. Al-Mutairi N. 308-nm excimer laser for the treatment of alopecia areata. Dermatol Surg 2007;33:1483-7.

23. Ohtsuki A, Hasegawa T, Ikeda S. Treatment of alopecia areata with 308-nm excimer lamp. J Dermatol 2010;37:1032-5.

24. Al-Mutairi N. 308-nm excimer laser for the treatment of alopecia areata in children. Pediatr Dermatol 2009;26:547-50.

25. Byun JW, Moon JH, Bang CY, Shin J, Choi GS. Effectiveness of 308-nm Excimer Laser Therapy in Treating Alopecia Areata, Determined by Examining the Treated Sides of Selected Alopecic Patches. Dermatology 2015;231:70-6.

26. Manstein D, Herron GS, Sink RK, Tanner H, Anderson RR. Fractional photothermolysis: a new concept for cutaneous remodeling using microscopic patterns of thermal injury. Lasers Surg Med 2004;34:426-38.

27. Yoo KH, Kim MN, Kim BJ, Kim CW. Treatment of alopecia areata with fractional photothermolysis laser. Int J Dermatol 2010;49:845-7.

28. Eckert MM, Gundin NL, Crespo RL. Alopecia areata: good response to treatment with fractional laser in 5 cases. J Cosmo Trichol 2016;2:108.

29. Kim WS, Lee HI, Lee JW, Lim YY, Lee SJ, Kim BJ, et al. Fractional photothermolysis laser treatment of male pattern hair loss. Dermatol Surg 2011;37:41-51.

30. Lee GY, Lee SJ, Kim WS. The effect of a $1550 \mathrm{~nm}$ fractional erbium-glass laser in female pattern hair loss. J Eur Acad
Dermatol Venereol 2011;25:1450-4.

31. Issa MC, Pires M, Silveira P, Xavier de Brito E, Sasajima C. Transepidermal drug delivery: a new treatment option for areata alopecia? J Cosmet Laser Ther 2015;17:37-40.

32. Lee WR, Shen SC, Aljuffali IA, Li YC, Fang JY. Erbiumyttrium-aluminum-garnet laser irradiation ameliorates skin permeation and follicular delivery of antialopecia drugs. J Pharm Sci 2014;103:3542-52.

33. Tsai TH, Jee SH, Chan JY, Lee JN, Lee WR, Dong CY, et al. Visualizing laser-skin interaction in vivo by multiphoton microscopy. J Biomed Opt 2009;14:024034.

34. Mester E, Szende B, Gärtner P. The effect of laser beams on the growth of hair in mice. Radiobiol Radiother (Berl) 1968;9:621-6.

35. Avci P, Gupta GK, Clark J, Wikonkal N, Hamblin MR. Low-level laser (light) therapy (LLLT) for treatment of hair loss. Lasers Surg Med 2014;46:144-51.

36. Leavitt M, Charles G, Heyman E, Michaels D. HairMax LaserComb laser phototherapy device in the treatment of male androgenetic alopecia: a randomized, double-blind, sham device-controlled, multicentre trial. Clin Drug Investig 2009;29:283-92.

37. King LE Jr, Silva KA, Kennedy VE, Sundberg JP. Lack of response to laser comb in spontaneous and graft-induced alopecia areata in C3H/HeJ mice. J Invest Dermatol 2014;134: 264-6.

38. Gupta AK, Daigle D. The use of low-level light therapy in the treatment of androgenetic alopecia and female pattern hair loss. J Dermatolog Treat 2014;25:162-3.

39. Waiz M, Saleh AZ, Hayani R, Jubory SO. Use of the pulsed infrared diode laser $(904 \mathrm{~nm})$ in the treatment of alopecia areata. J Cosmet Laser Ther 2006;8:27-30.

40. Abdelhalim NM. Eefficacy of low level laser therapy in the treatment of alopecia areata. Int J Physiother Res 2014;2:460-5.

41. Yamazaki M, Miura Y, Tsuboi R, Ogawa H. Linear polarized infrared irradiation using Super Lizer is an effective treatment for multiple-type alopecia areata. Int J Dermatol 2003;42:73840.

42. Yalici-Armagan B, Elcin G. The effect of neodymium: yttrium aluminum garnet and fractional carbon dioxide lasers on alopecia areata: a prospective controlled clinical trial. Dermatol Surg 2016;42:500-6.

43. Shapiro J, Madani S. Alopecia areata: diagnosis and management. Int J Dermatol 1999;38:S19-24. 